\title{
Dilepton measurements with HADES
}

\author{
Christian Müntz ${ }^{* \dagger}$ \\ J. W. Goethe-Universität Frankfurt \\ E-mail: c.muentz@gsi.de
}

\begin{abstract}
HADES is the only operating dielectron spectrometer in the energy regime 1-2 AGeV. The physics program aims at a systematic investigation of dielectron production in heavy ion as well as elementary and pion-induced reactions.

This contribution highlights recent results on electron pair production in ${ }^{12} \mathrm{C}+{ }^{12} \mathrm{C}$ collisions at an incident energy of $2 \mathrm{AGeV}$ with HADES. The measured pair production probabilities span over five orders of magnitude. Dalitz decays of $\pi^{0}$ and $\eta$ account for all the yield up to $0.15 \mathrm{GeV} / \mathrm{c}^{2}$, but for only about $50 \%$ above this invariant mass. The excess yield compared to the hadronic cocktail between the $\pi^{0}$-Dalitz and the $\rho / \omega$ invariant-mass region is in agreement with the former DLS result if one assumes that it scales with beam energy like pion production. Preliminary results from ${ }^{12} \mathrm{C}+{ }^{12} \mathrm{C}$ collisions at an incident energy of $1 \mathrm{AGeV}$ support this scenario.
\end{abstract}

Critical Point and Onset of Deconfinement - 4th International Workshop July 9 - 13, 2007

Darmstadt, Germany

\footnotetext{
*Speaker.

${ }^{\dagger}$ for the HADES Collaboration
} 


\section{Contents}

1. The mission of HADES 2

2. The HADES setup 2

3. Experimental results $\quad 4$

4. Summary and outlook 6

\section{The mission of HADES}

The properties of hot and dense hadronic matter represent a key problem in heavy- ion physics, with far-reaching implications for other fields such as the physics of compact stars. They are governed by non-perturbative QCD. Models predict, however, that hadron properties, such as mass and lifetime, depend on the temperature and density of the surrounding nuclear medium. While some hadronic many-body calculations give a broadening of the meson in-medium spectral function, other approaches predict dropping meson masses related to the restoration of chiral symmetry [1]. The experimental access is difficult as well, since the life time of the dense phase is extremely short, and most of the probes suffer from final state interactions, substantially masking the primary information. Dileptons $\left(\mu^{+} \mu^{-}\right.$or $\left.e^{+} e^{-}\right)$from decays of short-lived resonances produced inside the hadronic matter created in the course of (ultra)-relativistic heavy-ion collisions, however, are considered to represent the most direct probe, and dedicated experiments and analyses, e.g. CERES and NA60 at CERN SPS, and PHENIX at RHIC [3], are presently promoted. In the 1-2 AGeV incident energy regime the small branching ratios into the dielectron channel adds a particular experimental difficulty. The DLS collaboration found unexpectedly large electron-pair yields in $\mathrm{C}+\mathrm{C}$ and $\mathrm{Ca}+\mathrm{Ca}$ collisions [4] which cannot be described satisfactorily within the various scenarios proposed for possible changes of the in-medium spectral functions [1,5]. Indeed, the pair yields in the invariant-mass range between 0.15 and $0.6 \mathrm{GeV} / \mathrm{c}^{2}$, i.e. just below the $\rho$ meson pole mass, still remain to be explained [6]. Resolving this unsatisfactory situation by means of a high-accuracy and systematic investigation of dielectron production in heavy ion as well as elementary reactions was the primary motivation to set up the $2^{\text {nd }}$-generation experiment HADES

\section{The HADES setup}

The High-Acceptance DiElectron Spectrometer HADES at GSI, Darmstadt, is presently the only dilepton spectrometer operating in the energy regime of 1-2 AGeV, succeeding the DLS spectrometer at Bevalac [4]. The HADES spectrometer, described in detail in [2], consists of a 6-coil toroidal magnet centered on the beam axis and six identical detection sections located between the 
coils, covering polar angles between $18^{\circ}$ and $85^{\circ}$, see figure 1 where a cross section of the detector setup is depicted. In the measurement ${ }^{12} \mathrm{C}+{ }^{12} \mathrm{C}$ at $2 \mathrm{AGeV}$, each sector was composed of a gaseous Ring-Imaging Cherenkov (RICH) detector, two planes of Mini-Drift Chambers (MDC-I and MDCII) for track reconstruction between the RICH and the magnetic field, and a Time-Of-Flight wall (TOF/TOFino) supplemented at forward polar angles with Pre-SHOWER chambers. The interaction time was obtained from a fast diamond start detector located upstream of the target. In the following experiments $\left({ }^{12} \mathrm{C}+{ }^{12} \mathrm{C}\right.$ at $1 \mathrm{AGeV},{ }^{40} \mathrm{Ar}+\mathrm{KCl}$ at $1.75 \mathrm{AGeV}$ and elementary reactions) the tracking system was bit by bit completed with two planes of drift chambers between the magnetic field and the time-of-flight system, aiming at maximum momentum resolution in all six sectors. Hence, HADES provides a large and smooth dielectron acceptance of about $30 \%$. Recently, the Forward Hodoscope was added under polar angels smaller than $7^{\circ}$, which serves for tagging pn reactions in d-induced reactions and which will provide information on the reaction plane orientation in future heavy ion runs. Besides solid state targets, a liquid hydrogen target is available for the physics program regarding elementary reactions. For $\mathrm{C}+\mathrm{C}(2 \mathrm{AGeV})$ the data readout was

Figure 1: Cross section of the HADES setup, with tracks of charged particles, including an electron which triggers Cherenkov light in the RICH and an electromagnetic shower in the Pre-Shower detector in the upper sector. The beam is coming from the left, the RICH diameter amounts to $1.6 \mathrm{~m}$, see text for details.

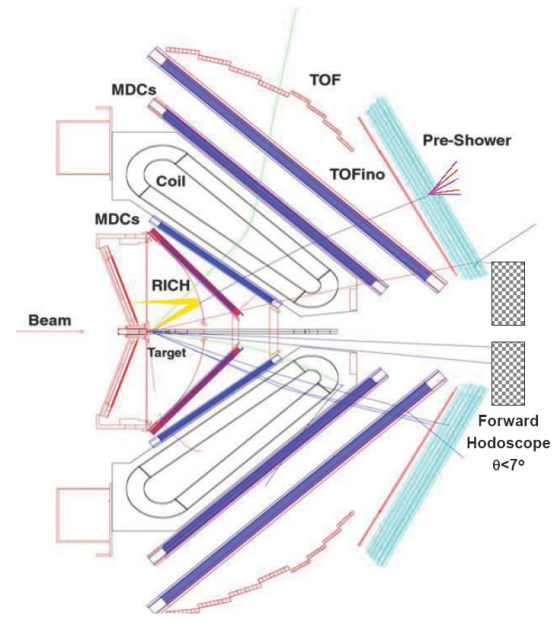

started by a first-level trigger (LVL1) decision, requiring a charged-particle multiplicity $M U L \leq 4$ in the TOF/TOFINO detectors, accepting $60 \%$ of the total cross section. It was followed by a second-level trigger (LVL2) requesting at least one electron track. With this trigger condition, a 10 -fold pair enrichment at a pair efficiency of $\leq 92 \%$ was achieved. Furthermore, the LVL2 introduced no bias on the shapes of measured pair distributions, as checked by a direct comparison to the unbiased LVL1 events. Uncorrelated electrons from $\pi^{0} \rightarrow \gamma \gamma$ decays followed by photon conversion and/or from $\pi^{0} \rightarrow e^{+} e^{-} \gamma$ Dalitz decays form most of the combinatorial background (CB). They are rejected efficiently by applying conditions on the opening angle $\theta_{e+e-}>9^{\circ}$ of the reconstructed track segments, removing $95 \%$ of the conversion pairs while reducing the dielectron signal with $M_{e e}>0.15 \mathrm{GeV} / \mathrm{c}^{2}$ by less than $10 \%$. Here, all numbers given are valid for the $2 \mathrm{AGeV}$ ${ }^{12} \mathrm{C}+{ }^{12} \mathrm{C}$ run. For further details, especially regarding the analysis, determining the combinatorial background as well as systematical errors, see [7, 2]. 


\section{Experimental results}

Figure 2a shows the $M_{e e}$ distribution of the signal pairs after efficiency correction and normalization to the average number of charged pions $N_{\pi}=\frac{1}{2}\left(N_{\pi^{+}}+N_{\pi^{-}}\right)$. The charged pion yield was measured in the HADES acceptance [2] and extrapolated to the full solid angle. The extrapolation takes into account measured angular distributions, found to be in agreement with UrQMD calculations [8]. The obtained pion multiplicity per number of participating nucleons $M_{\pi} / A_{\text {part }}=$ $0.137 \pm 0.015\left(A_{\text {part }}=9.0\right)$ agrees with previous measurements of charged and neutral pions [9] within the quoted error of $11 \%$. The error represents an overall normalization error, dominated by systematic uncertainties in the acceptance and efficiency corrections of the charged-pion analysis. The systematical uncertainties for the dielectron yield from the efficiency correction and the CB subtraction add up quadratically to a nearly constant error of $18 \%$. A pair cocktail (cocktail A)

Figure 2: (a) Efficiency- and background-corrected $e^{+} e^{-}$ invariant-mass distribution for $\theta_{e+e-}>9^{\circ}$ (symbols) compared to a thermal dielectron cocktail of free $\pi^{0}, \eta$ and $\omega$ decays (cocktail A, solid line), as well as including $\rho$ and $\Delta$ resonance decays (cocktail $\mathrm{B}$, long-dashed line). Only statistical errors are shown. (b) Ratio of data and cocktail A (dots), compared to ratios of various model calculations and cocktail $\mathrm{A}$ as a function of the invariant mass. All calculations have been filtered and folded with the HADES acceptance and mass resolution. Statistical and systematic errors of the measurement are shown as vertical and horizontal lines, respectively. The overall normalization error of $11 \%$ is depicted by the shaded area.

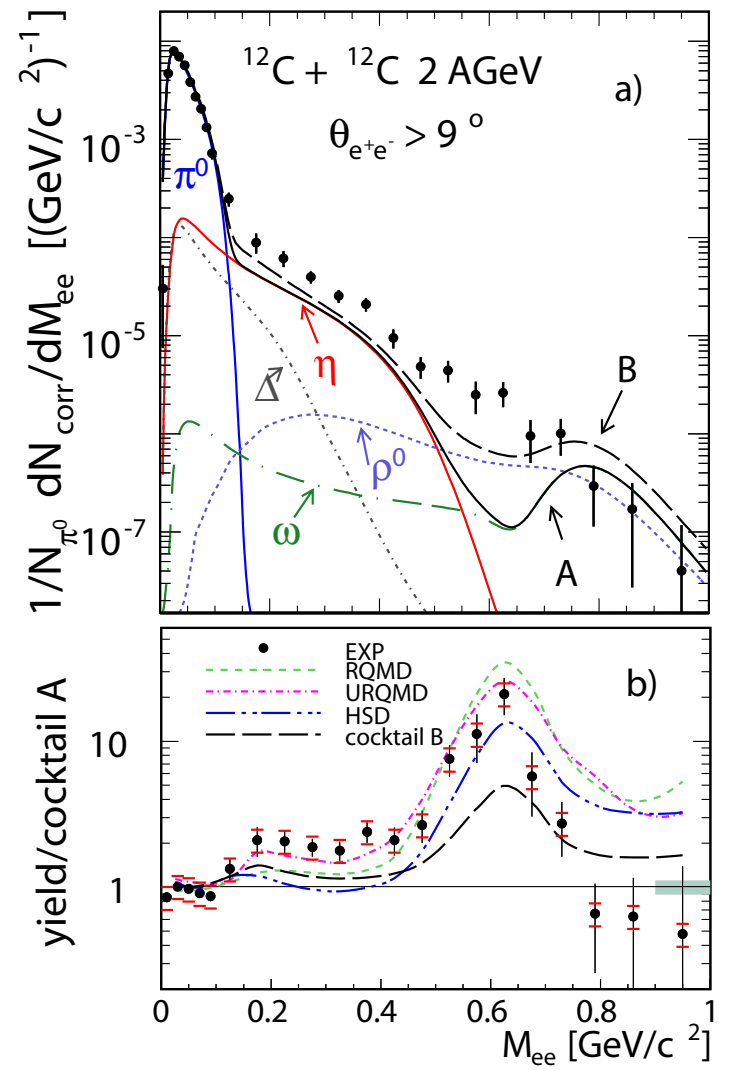

was calculated from free $\pi^{0}, \eta$ and $\omega$ meson decays only to represent all contributions emitted after the chemical freeze-out of the fireball and compared to the resulting $M_{e e}$ distribution (figure 2a). While $\pi^{0}$ and $\eta$ sources are directly constrained by data [9], the production rate of the $\omega$ meson is taken from a $m_{\perp}$-scaling ansatz [10]. In the HADES event generator PLUTO [11] meson production was modeled assuming emission from a thermal source with a temperature $T=80 \mathrm{MeV}$, without a radial expansion velocity. The anisotropic angular distribution, obtained from the charged-pion analysis, was used for the $\pi^{0}$ mesons. While experimental data and the 
simulated cocktail A are in good agreement in the $\pi^{0}$ region, the cocktail undershoots the data for $M>0.15 \mathrm{GeV} / \mathrm{c}^{2}$. Decays of short-lived resonances (as $\rho, \Delta(1232)$ ), excited in the early phase of the collision, will additionally contribute here. Assuming that the $\Delta$ yield scales with the $\pi^{0}$ yield and, the $\Delta^{0,+} \rightarrow N e^{+} e^{-}$decays were added to the cocktail, using a calculated decay rate [12]. Furthermore, the $\rho$ meson was treated in analogy to the $\omega$ meson. For the latter broad resonance $\left(\Gamma_{\rho}=150 \mathrm{MeV}\right), m_{\perp}$ scaling and available phase space strongly enhances the low-mass tail, resulting in the skewed shape visible in figure 2a. Comparing the full thermal cocktail (cocktail B) with the data, the simulated yield above $0.15 \mathrm{GeV} / \mathrm{c}^{2}$ is now increased and the high-mass region is populated with dielectrons from $\rho \rightarrow e^{+} e^{-}$decays, but the calculation still falls short of reproducing the data. In figure $2 \mathrm{~b}$ the ratio of the data and cocktail $\mathrm{A}$ is shown. In the intermediate mass range of $0.15-0.50 \mathrm{GeV} / \mathrm{c}^{2}$, the enhancement factor above the dominant $\eta$ contribution amounts to $F(2.0)=2.1 \pm 0.2$ (stat) $\pm 0.3($ sys $) \pm 0.4(\eta)$. The third error $(\eta)$ indicates the errors resulting from the quoted error in the measured $\eta$ multiplicity with TAPS [9].

The analysis of the $1 \mathrm{AGeV}{ }^{12} \mathrm{C}+{ }^{12} \mathrm{C}$ run is presently being concluded. Hence, only preliminary data can be shown and discussed. Comparing the acceptance- and efficiency-corrected invariant mass spectrum measured by HADES to the corresponding DLS spectrum [4], by extrapolating to the DLS phase space, an agreement within the statistical and systematical errors can be reported [13]. In comparison to the $2 \mathrm{AGeV}$ invariant mass spectrum the excess yield is much more pronounced, see figure 3 , yielding a preliminary enhancement factor above the dominant $\eta$ contribution of $F(1.0)=7.0 \pm 0.6($ stat $) \pm 1.1($ sys $) \pm 2.0(\eta)$. For generating cocktail A (1 AGeV) representing the long-lived mesons, a freeze-out temperature of $\mathrm{T}=55 \mathrm{MeV}$ was assumed.

Figure 3: Ratio of data (1 AGeV and $2 \mathrm{AGeV}$ incident energy) and the corresponding cocktail A of dielectrons originating from long-lived mesons as a function of the invariant mass, see text. The overall normalization error of $11 \%$ is depicted by the shaded area.

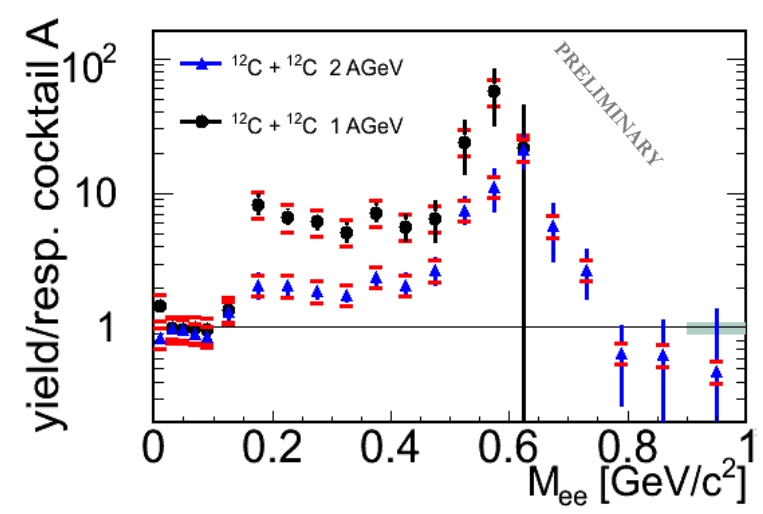

Employing the $\eta$-yield measured by TAPS [9] at 1.04 and $2 \mathrm{AGeV}$ the preliminary ratio of the absolute excess yields $Y_{\text {exc }}$ in ${ }^{12} \mathrm{C}+{ }^{12} \mathrm{C}$ reactions at both energies can be deduced: $Y_{\text {exc }}^{2 A G V} / Y_{\text {exc }}^{1 \mathrm{AGeV}}$ $=2.8 \pm 0.2($ stat $) \pm 1.0($ sys $) \pm 1.0(\eta)$. It is interesting to note that the energy scaling of the excess yield is similar to that of the pion production and does not follow the energy scaling of $\eta$ mesons [9]. 


\section{Summary and outlook}

HADES was set up at SIS/GSI Darmstadt to study dielectron production in heavy ion as well as elementary and pion-induced reactions in the energy regime of 1-2 $\mathrm{AGeV}$ in a very systematic way, with quality data. This paper highlights final results on ${ }^{12} \mathrm{C}+{ }^{12} \mathrm{C}$ collisions at $2 \mathrm{AGeV}$ incident energy, and their comparison to the preliminary results from $1 \mathrm{AGeV}$. In both data sets a significant dielectron excess yield above the thermal pair cocktail from decaying long-lived mesons was found. A quantitative analysis of this excess confirms the results reported by the DLS collaboration at $1 \mathrm{AGeV}$. The excess energy dependence is similar to the one of pions. This observation suggests the importance of baryonic resonances for the origin of the excess yield. It also demonstrates the need of a systematic investigation of elementary reactions as input for models, presently being pursued by the HADES collaboration. First preliminary results are available [14], focusing on the role of bremsstrahlung by comparing pp with pn (d-induced) reactions at $1.25 \mathrm{GeV}$ and hunting vector mesons in pp reactions at $3.5 \mathrm{GeV}$. Regarding the heavy ion program data from $\mathrm{Ar}+\mathrm{KCl}$ at $1.75 \mathrm{AGeV}$ are presently being analyzed, and HADES is facing a major upgrade by installing multi-gap timing RPC detectors and replacing central DAQ components to cope with the high multiplicities in $\mathrm{Au}+\mathrm{Au}$ reactions.

\section{References}

[1] R. Rapp and J. Wambach, Adv. Nucl. Phys. 25, 1 (2000).

[2] R. Schicker et al., Nucl. Instrum. Methods Phys. Res. A 380, 586 (1996), P. Salabura et al., Prog. Part. Nucl. Phys. 53, 49 (2004), Nucl. Phys. A 749, 150 (2005), and http://www-hades.gsi.de.

[3] see proceedings of this conference.

[4] R. J. Porter et al., Phys. Rev. Lett. 79, 1229 (1997).

[5] F. Klingl, N. Kaiser, and W. Weise, Nucl. Phys. A 624, 527 (1997), W. Peters et al., Nucl. Phys. A632, 109 (1998); B. Friman and H. Pirner, Nucl. Phys. A 617, 496 (1997).

[6] W. Cassing and E. L. Bratkovskaya, Phys. Rep. 308, 65 (1999), K. Shekhter et al., Phys. Rev. C 68, 014904 (2003). [8] M. D. Cozma, C. Fuchs, E. Santini, A. Faessler, Phys. Lett. B 640, 150 (2006), C. Ernst et al., Phys. Rev. C 58, 447 (1998). P. Salabura et al., Prog.

[7] G. Agakichiev et al., Phys. Rev. Lett. 98, 052302 (2007).

[8] D. Schumacher, S. Vogel, M. Bleicher, nucl-th/0608041.

[9] TAPS: R. Averbeck et al., TAPS coll., Z. Phys. A 359, 65 (1997), R. Holzmann et al., Phys. Rev. C 56, R2920 (1997), KaoS: C. Sturm et al., Phys. Rev. Lett. 86, 39 (2001).

[10] E. L. Bratkovskaya, W. Cassing, and U. Mosel, Phys. Lett. B 424, 244 (1998).

[11] M. A. Kagarlis, GSI Report 2000-03 (2000), unpublished.

[12] C. Ernst et al., Phys. Rev. C 58, 447 (1998).

[13] Y. C. Pachmayer, PhD thesis in preparation, University of Frankfurt, R. Holzman, private communications, to be submitted to Phys. Lett. B.

[14] P. Salabura et al., Progress in Particle and Nuclear Physics 53, 49 (2004), and the HADES proposal for experiment S301 at SIS/GSI Darmstadt 